\title{
Interpretation of desorption measurements For high energy beams at RHIC
}

\author{
U. Iriso, P. He, H.C. Hseuh, H. Huang, V. Ptitsyn, L. Smart, \\ P. Thieberger, D. Trbojevic, and S.Y. Zhang, \\ E. Mustafin and G. Rumolo
}

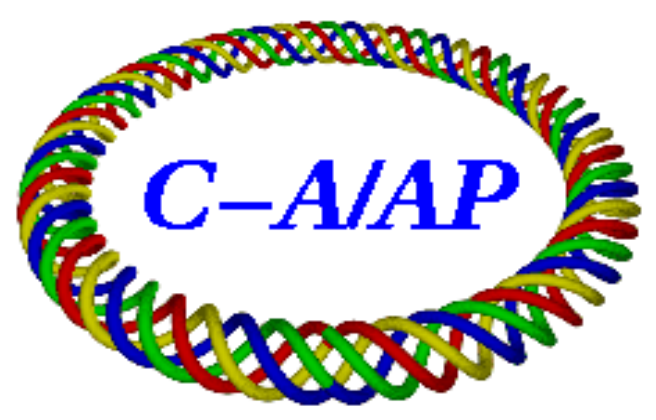

Collider-Accelerator Department Brookhaven National Laboratory Upton, NY 11973 
$\mathrm{CAD} / \mathrm{AP} / 178$

November 2, 2004

\title{
Interpretation of desorption measurements for high energy beams at RHIC
}

\author{
U. Iriso, P. He, H.C. Hseuh, H. Huang, V. Ptysin, L. Smart, \\ P. Thieberger, D. Trbojevic, and S.Y. Zhang *, E. Mustafin, and G. Rumolo ${ }^{\dagger}$
}

\begin{abstract}
During Run-3 (2002/2003), several pressure rise events occurred in the ramping process, when about $5 \%$ of the beam was lost. High energy stray ions lost at grazing angles were found to be a possible source for this vacuum degradation. In order to study this phenomenon, the beam was deliberately steered towards the wall of the beam pipe at controlled incident angles. From the analysis of the pressure evolution, the desorption coefficient for high energy gold ions (10 GeV energies) is inferred. In the following, we describe a method to dynamically interpret these pressure bumps, which does not successfully reproduce the results unless we assume large surface residence times. The desorption coefficients obtained with this method are finally compared with results from other analysis, which is independent of any gas-conserving mechanism.
\end{abstract}

\section{Introduction}

During Run-3 (2002/2003), several pressure rise events occurred in the ramping process, when about $5 \%$ of the beam was lost [1]. These pressure rises were found to be more pronounced in the presence of two beams, i.e. in the Interaction Regions, producing high experimental backgrounds and limiting machine operation. An exponential dependence on the total beam intensity (two beams) was found for this pressure rise [2]. High energy stray ions lost at grazing angles were found to be a possible source for this vacuum degradation, and analysis from these events predicted high desorption coefficients $\left(\eta_{e f}\right)$ of around $10^{7}$ molecules/ion for angles in the mrad range [3, 4]. Figure 1 shows an overview of heavy-ion induced desorption data obtained at different laboratories and presented at [4], where the RHIC point corresponding to gold ions $\left(A u^{79+}\right)$ at $10 \mathrm{GeV}$ is obtained after the above mentioned discussion. In order to confirm this result and study this phenomenon, the beam was deliberately steered towards the wall of the beam pipe at grazing incident angles. From the analysis of the pressure evolution, the desorption coefficient for gold ions at $10 \mathrm{GeV}$ is inferred.

The experiment was performed in several locations of the ring, generating about 200 sets of data. In this note, we look for a valid physical model to interpret one of the typical pressure bumps. The experiment is sketched in Fig. 2: using the kicker at the end of the cold bore, the beam is dumped at the chamber wall in region "yo12". The target location is controlled using the orbit application. After the injected beam hits the wall, the pressure increases due to the desorption of molecules from the pipe wall, and the vacuum pumps reestablish equilibrium in a decay time of, typically, around ten seconds. Figure 3 shows one of these experiments, which took place in a region of the ring labelled "yo12". Each bump corresponds to a shot of four bunches of injected beam dumped at a single beam pipe spot. Each shot hits a different location of the chamber wall.

The following sections show that the main difficulty is not to interpret the pressure increase, but to reproduce the time pressure evolution. Section 3 describes the analysis of a single pressure bump in yo12 including the dynamic process. It is shown that after the introduction of some physical parameters (sticking wall coefficient and surface resident time), the dynamics of the process is successfully reproduced. Nonetheless, the lack of knowledge of the gas composition (no Residual Gas Analyzers are installed in the experiments area), and the large number of unknown surface parameters give a large number of degrees of freedom to the dynamic process. The height of the pressure rise itself is mainly due to the desorption coefficient, while

*BNL, Upton, NY - 11973, USA

${ }^{\dagger}$ GSI, Darmstaadt, Germany 


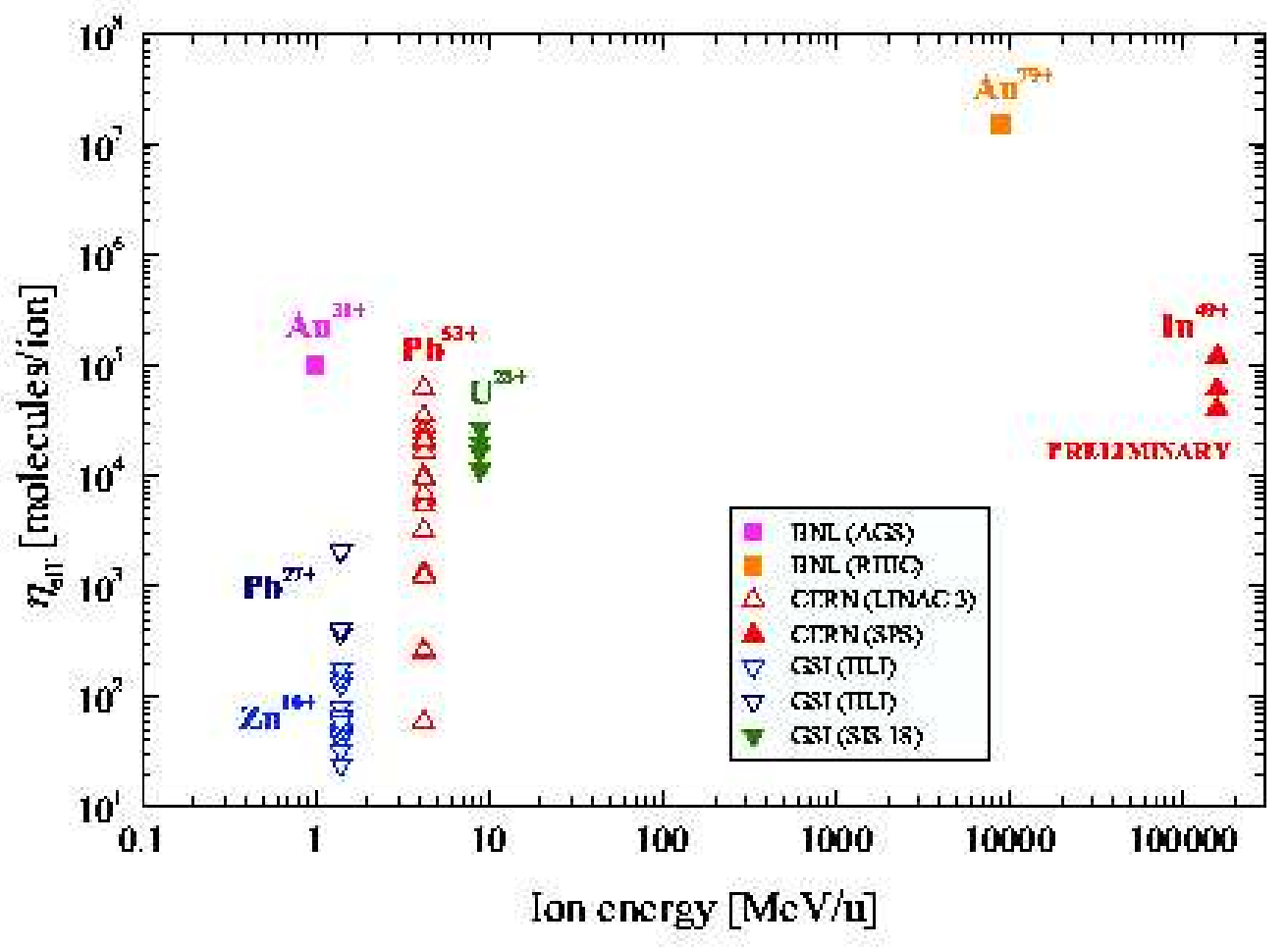

Figure 1: Overview of heavy-ion induced desorption data, obtained at BNL, CERN, and GSI. The ion impact angles (perpendicular, mrad, $\mu \mathrm{rad}$ ) of the experiments are different. Results reported at Ref. [4].

the rest of the parameters have a second (but non-negligible) influence. Since the goal of these experiments is to come up with a significant value of the desorption coefficient $\left(\eta_{e f}\right)$, we focus the study on a scan of this parameter while keeping the others inside reasonable ranges to obtain an optimum reproducibility of the pressure bump.

\section{Static pressure at yo12}

Table 1 lists the vacuum elements in the RHIC section "yo12". We study this particular section because it is one of the cleanest in terms of elements introduced there for beam diagnostics and/or control. Except for the polarimeter, the rest of the beam pipe $(34 \mathrm{~m})$ is made of baked stainless steel, with a roughly constant diameter $(12 \mathrm{~cm}$ ) (see Figure 2). Similar to the other sextants in RHIC, this section is followed at both ends by the arcs, which are held at superconducting temperatures $(4 \mathrm{~K})$. The $1.59 \mathrm{~m}$ long polarimeter is placed at the beginning of the beam pipe. It consists of several components with different materials: ceramic, alluminimium, and stainless steel.

Using the apertures of the vacuum tubes and pumping speeds of the pumps given in Table 1, the static pressure is calculated solving the equation in [5] for the the linear outgassing rate $q$ in $t o r r . l / s / m$ along the longitudinal position, $z$ :

$$
c(z) \frac{\partial^{2} P(z)}{\partial z^{2}}=-q(z),
$$

where $P(z)$ is the pressure along the longitudinal position. Expressing $P$ in torr, the units for the specific gas flow are torr $\cdot l /(s \cdot m)$, and the conductance $c(z)$ is calculated as

$$
c(z)=0.928 \cdot r^{3}(z) \sqrt{\frac{28}{M} \cdot \frac{T}{300}},
$$

where $r$ is the radius of the tube. Equation 2 expresses the conductance in $l \cdot \mathrm{m} / \mathrm{s}$ when $r$ is in $\mathrm{cm}$. $M$ 


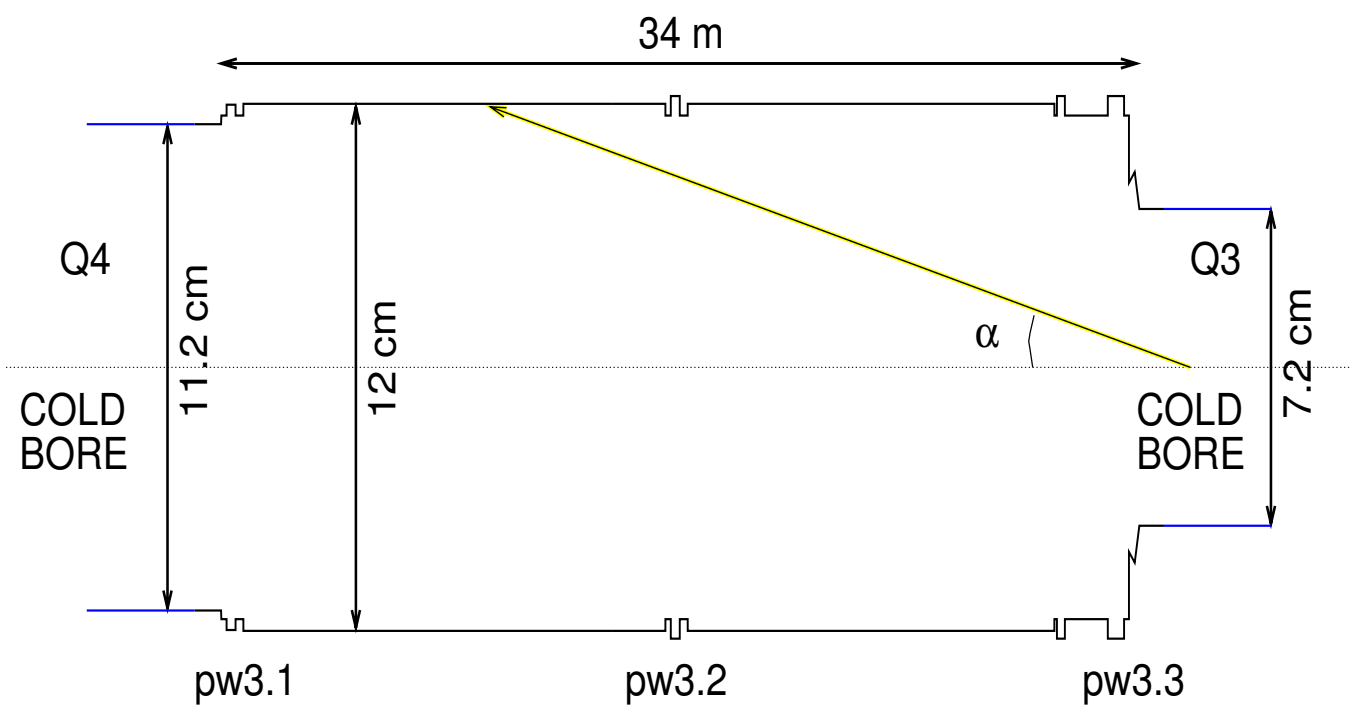

Figure 2: Layout of the experiment. The injected beam travels from pw3.3 towards pw3.1. At the edge of the cold bore (right side), the beam is stored at different angles to steer it towards the beam pipe wall.

and $T$ are the mass of the gas component under study and the temperature of the system, respectively. All calculations have been done for $\mathrm{CO}$ at room temperature $(\mathrm{M}=28, \mathrm{~T}=293 \mathrm{~K})$.

The static pressure profile was calculated for the apertures of the vacuum tubes and pumping speeds given in Table 1. The values for the pumping speeds take into account the conductance and cryopumping from the cold bore, which is only $1 \mathrm{~m}$ away from the pumps. This is why the pumps at "pw3.1" and "pw3.3" have a larger pumping speed than "pw3.2". The program used is $V A S T$, written by E. Mustafin [6]. The final pressure profile under static conditions is seen in Fig. 4, top plot, while the beam pipe geometry is shown on the bottom part. In order to match the readings of the vacuum pumps "pw3.1", "pw3.2", and "pw3.3", the values of the thermal outgassing rate $K$ for the elements in the vacuum pipe have been adjusted within a reasonable range [5], depending on the material and on the gas specie $\left(K_{C O}\right.$ and $\left.K_{H_{2}}\right)$. In the stainless steel surface, these values are:

$$
K_{C O}=3.75 \cdot 10^{-14} \text { torr } \cdot \mathrm{l} / \mathrm{s} / \mathrm{cm}^{2} \text { for } \mathrm{CO} \text {; and } K_{\mathrm{H}_{2}}=7.5 \cdot 10^{-14} \mathrm{torr} \cdot \mathrm{l} / \mathrm{s} / \mathrm{cm}^{2} \text { for } \mathrm{H}_{2}
$$

in the stainless steel surface. In the polarimeter part, they are

$$
K_{C O}=1.24 \cdot 10^{-11} \text { torr } \cdot \mathrm{l} / \mathrm{s} / \mathrm{cm}^{2} \text { for CO; and } K_{H_{2}}=2.5 \cdot 10^{-11} \text { torr } \cdot \mathrm{l} / \mathrm{s} / \mathrm{cm}^{2} \text { for } H_{2} \text {. }
$$

The static pressure has been calculated for two different boundary conditions: given the pressure values at both ends (Fig. 4, top plot, red trace), and assuming zero flow at the end of both sections (Fig. 4, top plot, light blue trace). Both conditions give similar pressure profile, the agreement is acceptable for both ends (readings at "pw3.1" and "pw3.3"), but differ for "pw3.2". In order to adjust the pressure readings at "pw3.2", one can always slightly change the outgassing rate for the different parts of the beam pipe, which is reasonable since the bake out could not be carried out uniformily along the beam pipe. However, since the discrepancy is small and the pressure gauge readings have an accuracy of only about $30 \%$, further refinement makes no sense, and we prefer not to change the outgassing values if the material surface does not change. Except for the polarimeter, all the beam pipe is made of stainless steel. The errors in the pressure gauge readings and the slightly different outgassing values might be the reasons for this (small) discrepancy. The approximation of zero flow ("sealed ends") at both ends of the chamber (having considered larger effective pumping speed at pumps "pw3.1" and "pw3.3" due to the cryopumping) is used from here on for simplicity. 


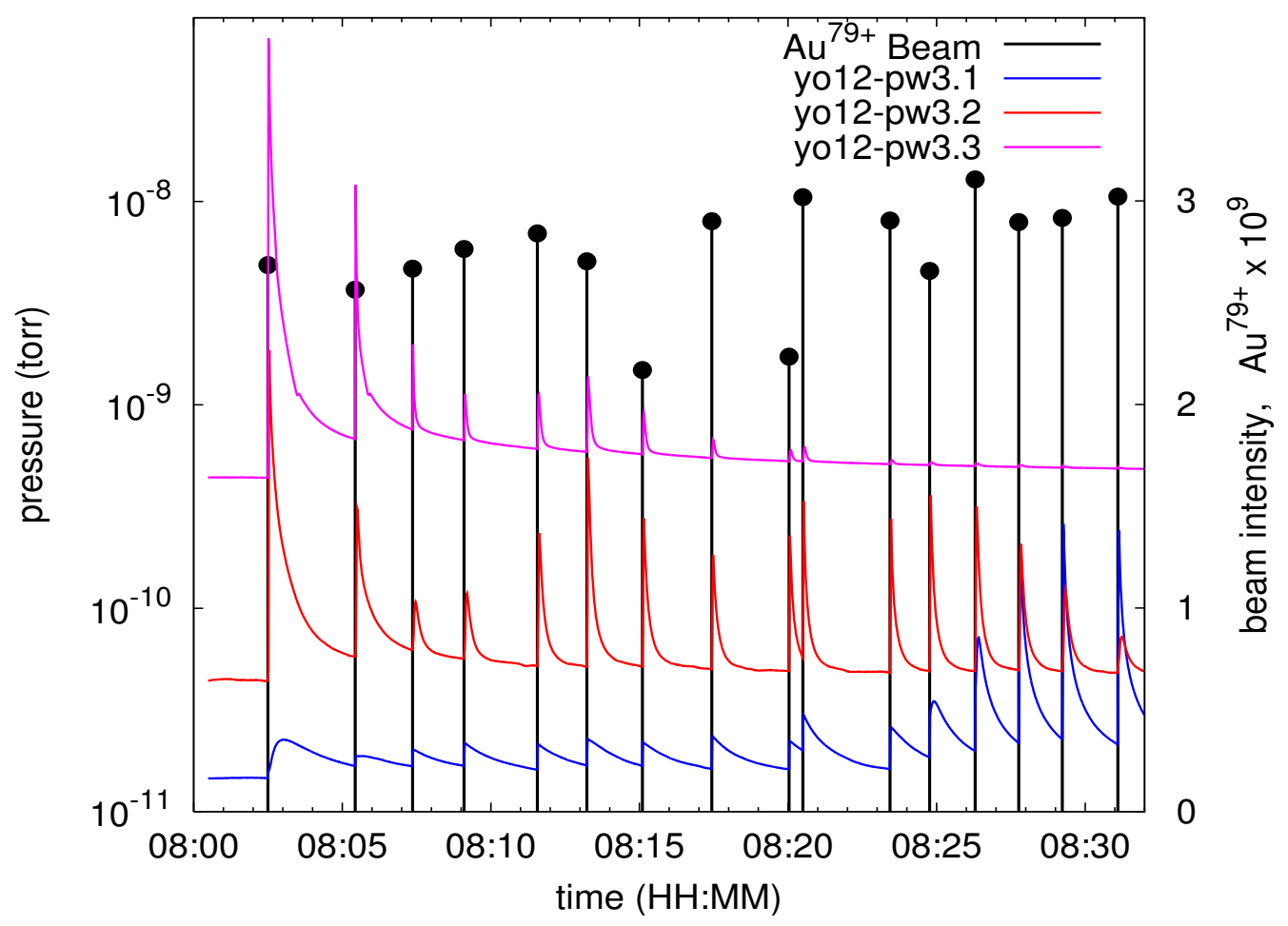

Figure 3: Scraping test on section "yo12". Each shot corresponds to four bunches, which are all dumped at a beam pipe location that changes for each shot. The total intensity of the four bunches is shown on the right axis of the figures (black pulses with dots), while the pressure readings of the different gauges in the beam pipe are shown on the left axis.

\section{Analysis of a single pressure bump}

\subsection{Dynamic pressure approach 1: The diffusion equation.}

The most natural way to tackle the time dependence pressure is using the diffusion equation [5]:

$$
v(z) \frac{\partial P(t, z)}{\partial t}-c(z) \frac{\partial^{2} P(t, z)}{\partial z^{2}}+s(z) P(t, z)=q(z),
$$

with $v(z)$ the linear volume (in $l / m$ ) and $s(z)$ the linear pumping speed. This equation has an analytical solution for a uniform and infinetely long vacuum tube:

$$
P(t, z)=\frac{Q}{\sqrt{4 \pi v c t}} e^{-\frac{v\left(z-z_{0}\right)^{2}}{4 c t}},
$$

where, $c$ is the conductance in $l \cdot m / s$, and $Q$ is the total amount of gas produced at $z_{0}$, in torr $\cdot l$. This does not give satisfactory results because the pressure expansion speed is much higher than measured. In other words, the maximum pressure value arrives at the pump position much faster than in the measurement. Assuming a bump at $t=0$ modeled with a delta function and at a given position $z_{0}$, Equation 4 has a maximum at:

$$
t=\frac{v\left(z-z_{0}\right)^{2}}{2 c}
$$

which gives the arrival time for the maximum pressure value. For the case under study, using $r=6 \mathrm{~cm}, v$ and $c$ become:

$$
\begin{aligned}
& v=\pi r^{2} ; \quad v=11 \quad l / m \\
& c=92.8 r^{3} \sqrt{(T / 300) \cdot(28 / M)} \quad ; \quad c=200 \quad l \cdot m / s .
\end{aligned}
$$




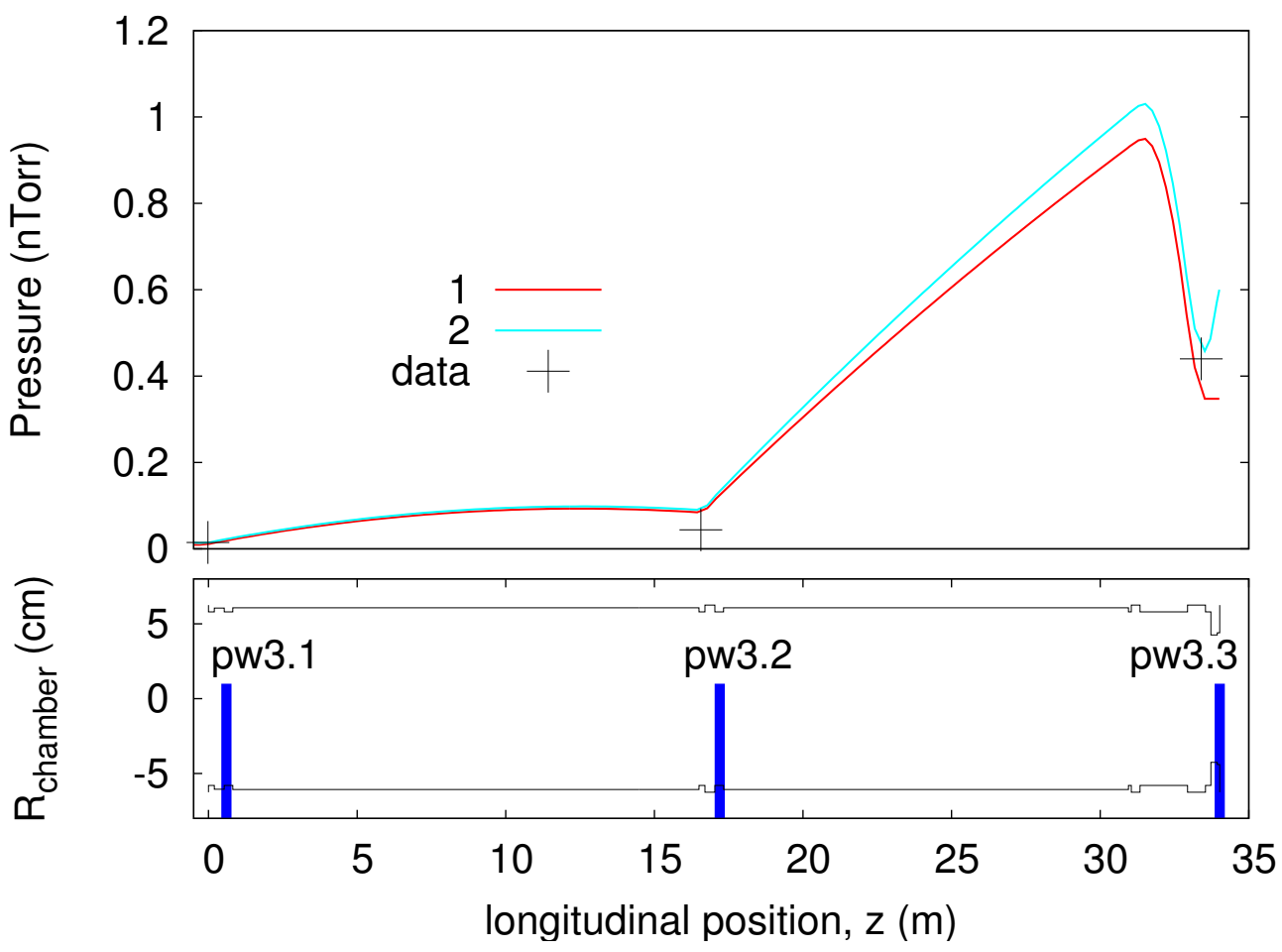

Figure 4: Vacuum pressure profile in static conditions (top plot) and aperture profile in section "yo12" (bottom plot). The blue bars in the bottom plot mark the presence of a vacuum pump. The asymmetry in the pressure is due to the presence of the polarimeter, which produces a larger thermal desorption rate than the rest of the beam pipe (stainless steel).

The arrival time is defined as the time at which the pressure gets its maximum in a location $z$, after a pressure bump is created at a location $z_{0}$ at time $t=0$. This time is shown in Fig. 5: the arrival times are in the order of seconds only for pressure bumps created at more than $6 \mathrm{~m}$.

Figure 6 shows the reproduction of a single shot using Eq. 3. Although one could find a reasonable agreement between the value of the maximum pressure for gauges at "pw3.3" and "pw3.2" (top and middle curve, respectively), the behaviour of "pw3.1" significantly differs. Moreover, one quickly notices that the dynamics of the process is not successfully reproduced. In general, the arrival time of the maximum pressure is smaller than what is measured. These mismatches must be either because Eq. 3 is incomplete, or because of problems with the vacuum readings. The vacuum readings might have an error bar of around $30 \%$, but this should not be a source of error for the time evolution: the time response of the cold cathode gauges has been measured to be about $100 \mathrm{~ms}$. In order to delay the arrival of the maximum pressure reading, we will introduce a second term in Eq. 4, to take into account the "surface residence time" of the molecules.

\subsection{Dynamic pressure approach 2: The surface resident time.}

In this approach, we make the assumption that molecules released from the vacuum chamber wall under the lost beam bombardment have a non-negligible surface residence time. This implies the introduction of one additional terms in Eq. 3:

$$
v(z) \frac{\partial P(t, z)}{\partial t}-c(z) \frac{\partial^{2} P(t, z)}{\partial z^{2}}+s_{1}(z) P(t, z)-s_{2}(z) P(t-\tau, z)=q(z) \quad .
$$

In this case, the term $s_{1} \cdot P(t, z)$ represents the sticking of the molecules at time $t$, and the term $s_{2}(z) \cdot P(t-\tau, z)$ represents the release of the molecules after their surface residence time $\tau$. According to Reference [7], the surface residence time for $\mathrm{CO}$ and $\mathrm{CO}_{2}$ is typically around $10^{-11} \mathrm{~s}$, although it can be even 10 orders of magnitude larger [9]. In any case, tens of miliseconds is small compared to the dynamic range of the process 
Table 1: List of vacuum elements in the RHIC beam pipe at "yo12", dimensions and pumping speed.

\begin{tabular}{lccc}
\hline \hline & diameter & length & pumping speed \\
unit & $\mathrm{cm}$ & $\mathrm{cm}$ & $\mathrm{l} / \mathrm{s}$ \\
\hline Gate Valve & 12.5 & 9.75 & - \\
Bellows & 11.6 & 19.50 & - \\
Pump "pw3.1" & 12.1 & 33.33 & 270 \\
5" pipe & 12.4 & 519.63 & - \\
5" pipe & 12.4 & 519.63 & - \\
Electron Detector & 12.4 & 23.93 & - \\
5" pipe & 12.4 & 504.95 & - \\
Bellows & 11.6 & 20.32 & - \\
Pump "pw3.2" & 12.1 & 33.33 & 166 \\
5" pipe & 12.1 & 519.63 & - \\
5" pipe & 12.1 & 519.63 & - \\
5" pipe & 12.1 & 323.10 & - \\
Gate Valve & 11.60 & 9.754 & - \\
Bellows & 12.5 & 29.26 & - \\
Polarimeter & 11.6 & 159.82 & - \\
Bellows & 12.5 & 26.98 & - \\
Pump "pw3.3" & 12.5 & 33.33 & 270 \\
Bellows & 11.6 & 18.08 & - \\
Transition & 8.5 & 21.60 & - \\
Gate Valve & 8.8 & 8.50 & - \\
\hline \hline
\end{tabular}

(some seconds). Therefore, the fourth element in Eq. 8 can be expanded as

$$
s_{2}(z) P(t-\tau, z)=s_{2}(z) P(t)-s_{2}(z) \tau \frac{d P(t)}{d t},
$$

and Eq. 8 becomes:

$$
\left[v(z)+s_{2} \tau\right] \frac{\partial P(t, z)}{\partial t}-c(z) \frac{\partial^{2} P(t, z)}{\partial z^{2}}+\left(s_{1}-s_{2}\right) P(t, z)=q(z),
$$

which in form is exactly the same as Eq. 3 but with different coefficients.

It would be natural to assume the same values for $s_{1}$ and $s_{2}$, i.e. all molecules which stick to the wall at time $t$ will be released in surface residence time $\tau$. Then the only parameter to fit in Eq. 10 is the coefficient $s_{2} \tau$. Unfortunately, predicted results do not reproduce the time evolution of the pressure bump, as can be seen on the left side of Fig. 7. The main difference is the slow pressure decay for this simulated case with respect to the measurement. This means that there is some pure linear pumping of the walls for the released molecules. Thus, we decide to investigate the case $s_{1} \neq s_{2}$, which is shown on the right hand side of Fig. 7 . The peak region of the measured pressure evolution at the pump position is more or less well fitted, but the tail of the pressure decay is not be well represented.

\subsection{Dynamic pressure approach 3: The two component gas.}

Our last approximation consists in assuming that the maximum of the pressure curves and the tails are due to two different type of molecules. Unfortunately, due to the lack of fast response Residual Gas Analyzers in the experimental area, we cannot identify the gas species involved in the process. With this assumption, one can arrive to an acceptable reproducibility (see Fig. 8).

It is worth mentioning that one could always get a better fit to the curve for pressure at "pw3.1" as well, but it would not make much sense because we used a rather rough assumption: the spread of surface residence times in the released gas is represented just by two components. 


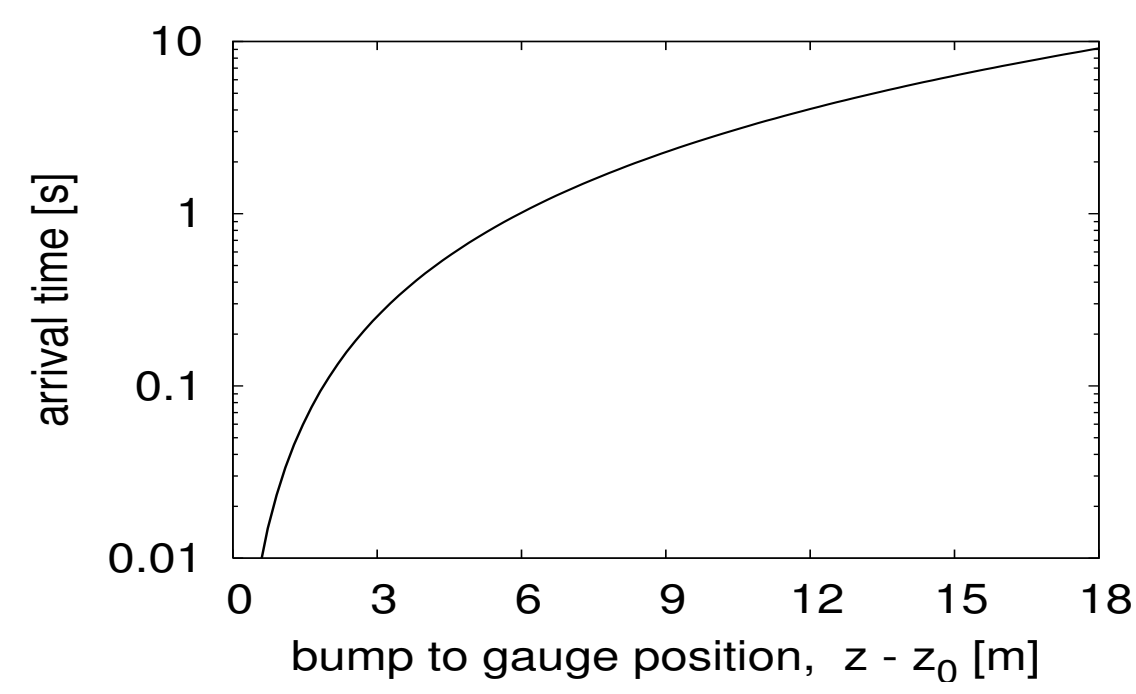

Figure 5: Arrival time for the maximum pressure to a gauge placed at a position $z$ from the pressure source.

Table 2: List of the different parameters used in the different approaches. In the last approach, the value in brackets shows the values corresponding to the second gas component taken in consideration.

\begin{tabular}{lcccc}
\hline \hline approach & $\eta_{e f}$ & $s_{2} \tau$ & $s_{1}-s_{2}$ & Figure \# \\
- & molecules/ion & $\mathrm{l} / \mathrm{m}$ & $\mathrm{l} / \mathrm{s} / \mathrm{m}$ & - \\
\hline Sect. 3.1: The diffusion equation & $0.3 \cdot 10^{4}$ & - & - & 6 \\
Sect. 3.2: The surface residence time, $s_{1}=s_{2}$ & $1 \cdot 10^{4}$ & 40 & - & 7 , left \\
Sect. 3.2: The surface residence time, $s_{1} \neq s_{2}$ & $7 \cdot 10^{4}$ & 70 & 30 & 7 , right \\
Sect. 3.3: The two component gas & $5 \cdot 10^{4}\left(2 \cdot 10^{4}\right)$ & $55(100)$ & $30(15)$ & 8 \\
\hline \hline
\end{tabular}

\subsection{Discussion}

A pumping speed of $s_{1}-s_{2} \approx 20 \mathrm{l} / \mathrm{s} / \mathrm{m}$ can be explained by the surface adsorption of part of the molecules released by the beam impact. The analysis requires a small calculation for the surface residence time. The rms thermal speed of the molecules is:

$$
v_{t h}=\sqrt{\frac{3 k T}{m}},
$$

where $k$ is the Boltzman's constant, $T$ is the temperature, and $m$ is the mass of the gas taken into consideration. For $C O$ or dry air, this is $v_{t h} \approx 500 \mathrm{~m} / \mathrm{s}$. For a chamber diameter $d$, we can calculate the frequency of collisions with the chamber wall as:

$$
\omega \approx v_{t h} / d
$$

This expression yields a value of $\omega \approx 4000 \mathrm{~Hz}$ with $d=12 \mathrm{~cm}$. If molecules glue to the wall with probability close to one, and taking into account that $s_{\text {avg }} \approx 25 \mathrm{l} / \mathrm{s}$ for $C O$, the linear pumping speed is estimated as:

$$
s_{2}=s_{\text {avg }} \cdot \omega \quad \approx 10^{5} \mathrm{l} / \mathrm{s} / \mathrm{m} .
$$

Taking into account that we found $s_{2} \cdot \tau \approx 100 \mathrm{l} / \mathrm{m}$, one ends up with a surface residence time $\tau \approx 1 \mathrm{~ms}$. As stated earlier in the text, Ref. [7] gives surface residence times on the order of $10^{-11} \mathrm{~s}$ for $\mathrm{CO}$ and $\mathrm{CO}_{2}$. Only organic molecules (whose presence is rather unlikely in UHV systems) have time values around one ms. However, the residence time is a strong function of the temperature and the heat of adsorption, and can have large variations (even by 10 orders of magnitude, from $10^{-11} \mathrm{~s}$ to some seconds [9]). Experimental values in the literature also range a lot: $\mathrm{O}$. Grobner uses in Ref. [10] $\tau \approx 10^{-2} \mathrm{~s}$ for $H_{2}$ and $\tau \approx 10^{1} \mathrm{~s}$ for $\mathrm{CO}$ in order to explain the behaviour of pressure bumps in the ISR, whereas J.P. Hobson and J.W. Earnshow 


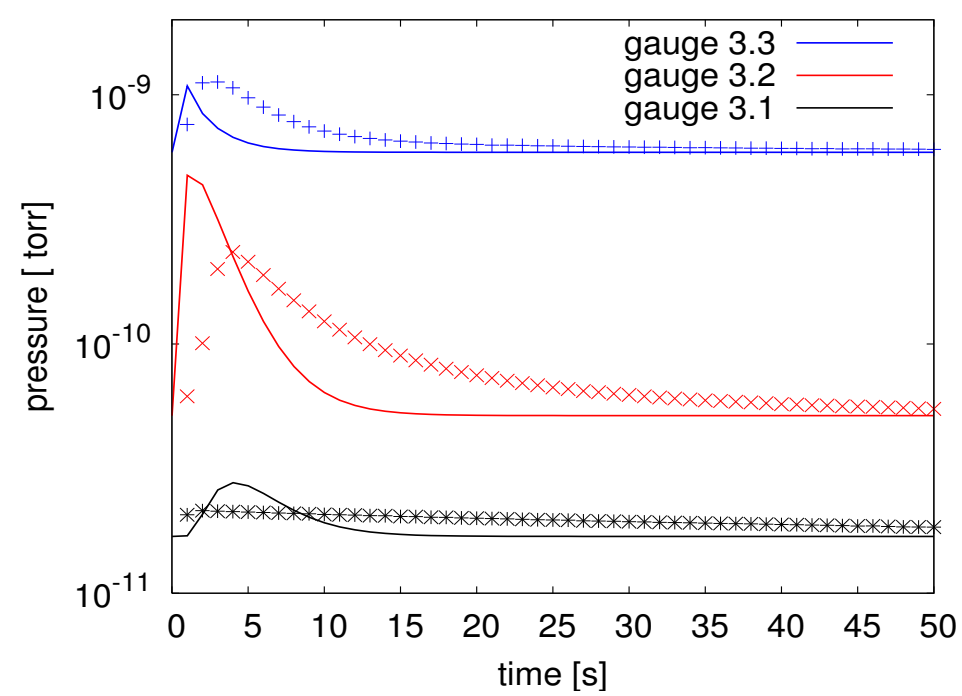

Figure 6: Measured vacuum pressure evolution (blue and red points) and reproduced behaviour using Eq. 3. Measurement and model differ for readings at all three gauges. Note that the reading at gauge "pw3.1" differs both in the maximum value and in the dynamic behaviour.

uses $\tau \approx 20 \mathrm{~ms}$ for CO on glass (see Eq. 75 at Ref. [11]). Thus the whole set of approaches looks physically reasonable, although perhaps surprising according to some of the existing literature. Nevertheless, interpretation of all the 200 pressure bumps at the different locations (and hence different surfaces) using the two gas approximation without having an RGA measure would be a nonsense calculation. We just wanted to emphasyze that by the use of this method, the measurements can be succesfully reproduced under these (reasonable) physical assumptions.

Therefore, the following sections focus only on the desorption coefficient using the aproximation explained in Section 3.2. In this case, the first priority is to reproduce the pressure height rather than the pressure time evolution, since this was the main purpose of the study, and one of the factors potentially limiting machine operation at RHIC.

\section{Experimental results}

We now focus on the accurate reproduction of the pressure bump according to Eq. 10, using a one component gas with $s_{1} \neq s_{2}$. This equation is solved by means of a computer program whose input parameters are:

- the gold ions beam intensity. The program assumes a beam impact on only half of the pipe transversal perimeter, that is, half a circle.

- the center of the beam impact location along the pipe, $z$ (longitudinally).

- the range of the beam impact. This parameter usually ranges between $0.5 \mathrm{~m}$ and $2 \mathrm{~m}$, according to a beam of $5 \mathrm{~mm}$ diameter impacting at angles between 1 and $5 \mathrm{mrad}$.

- the product $s_{2} \cdot \tau$, which mainly influences the delay of the time arrival (coefficient in the first element in Eq. 10).

- the substraction $\left(s_{1}-s_{2}\right)$, which mainly influences the decay in the tail of the pressure evolution (coefficient multiplying the third element in Eq. 10).

- the desorption coefficient $\eta_{e f}$, which influences the height of the pressure bump

The influence of these two coefficients $\left(s_{1} \cdot \tau\right.$ and $\left.\left(s_{1}-s_{2}\right)\right)$ is not only limited to the above mentioned effects. They also have an influence on the pressure height arriving at the gauge and, therefore, they also influence the final value of the desorption coefficient, $\eta_{e f}$. 

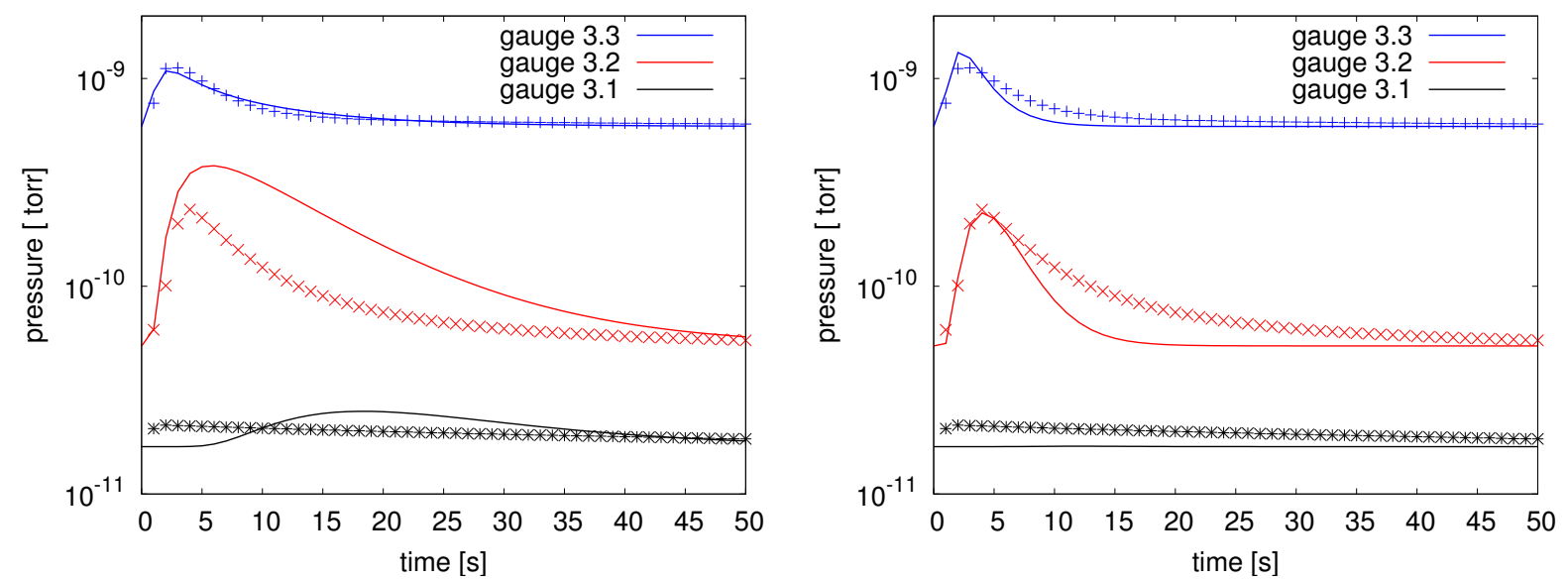

Figure 7: Pressure measurement (crosses) at the different gauges, and reproduced pressure behaviour (line traces) assuming the molecules have a non-negligible sticking coefficient but no linear pumping speed from the walls (left plot); whereas the right hand plot assumes both a sticking coefficient and a linear pumping speed from the beam pipe walls.

We now show the results and plots for each one of the 16 pressure bumps excited on February $25^{\text {th }} 2004$. Table 3 summarizes the parameter results found to reproduce the particular bump, and it shows the figure number where the result of the fit can be seen.

The angle is calculating by simple trigonometrics using the measured distance between the kicker and the beam impact, and the radius of the beam pipe. Since one of the input parameters is the position at which the beam impacts, we then have a measured angle (" $\alpha$ meas", in Table 3 ) corresponding to the calculation using the measured position; and a modeled angle (" $\alpha$ model", in Table 3 ) corresponding to calculation using the position used in our program. Assuming an error bar of $\pm 2 \mathrm{~mm}$ in the transverse beam position, the precision in the measured angle measurement is $\pm 1 \mathrm{mrad}$, consistent with the modeled angle.

Disregarding the two first measurements, which hit very close to the polarimeter region, the average value for the desorption coefficient for stainless steel is calculated to be $(3 \pm 2) \cdot 10^{4}$ molecules per impinging gold ion at $10 \mathrm{GeV}$, for angles that vary between 1 and 4 mrad.

Table 3: Results obtained for the 16 pressure bumps excited on February $25^{\text {th }} 2004$. The location is referred to gauge at "pw3.1".

\begin{tabular}{|c|c|c|c|c|c|c|c|c|}
\hline & $\begin{array}{l}\text { time } \\
\text { hh:mm }\end{array}$ & $\begin{array}{c}\text { intensity } \\
A u^{79+}\end{array}$ & $\begin{array}{c}\alpha, \text { meas } \\
\text { mrad }\end{array}$ & $\begin{array}{c}\alpha, \text { model } \\
\text { mrad }\end{array}$ & $\begin{array}{l}s_{2} \tau \\
\mathrm{l} / \mathrm{m}\end{array}$ & $\begin{array}{l}s_{1}-s_{2} \\
\mathrm{l} / \mathrm{s} / \mathrm{m}\end{array}$ & $\begin{array}{c}\eta_{e f} \\
\text { mlcs/ion }\end{array}$ & $\begin{array}{c}\text { Figure } \\
-\end{array}$ \\
\hline 1 & $08: 02$ & $2.60 \cdot 10^{9}$ & 4.0 & 4.0 & 70 & 15 & $1.0 \cdot 10^{6}$ & 11, left \\
\hline 2 & 08:05 & $3.75 \cdot 10^{9}$ & 3.7 & 3.7 & 60 & 28 & $2.0 \cdot 10^{5}$ & 11 , right \\
\hline 3 & 08:07 & $2.62 \cdot 10^{9}$ & 3.3 & 3.5 & 80 & 35 & $7.0 \cdot 10^{4}$ & 12 , left \\
\hline 4 & 08:09 & $2.71 \cdot 10^{9}$ & 3.1 & 3.3 & 70 & 35 & $5.0 \cdot 10^{4}$ & 12 , right \\
\hline 5 & 08:11 & $2.79 \cdot 10^{9}$ & 2.8 & 3.2 & 70 & 30 & $7.0 \cdot 10^{4}$ & 13, left \\
\hline 6 & $08: 13$ & $2.66 \cdot 10^{9}$ & 2.7 & 3.1 & 65 & 17 & $7.0 \cdot 10^{4}$ & 13 , right \\
\hline 7 & $08: 15$ & $2.12 \cdot 10^{9}$ & 2.5 & 3.0 & 80 & 20 & $5.0 \cdot 10^{4}$ & 14, left \\
\hline 8 & $08: 17$ & $2.82 \cdot 10^{9}$ & 2.3 & 2.9 & 70 & 20 & $1.5 \cdot 10^{4}$ & 14 , right \\
\hline 9 & $08: 20$ & $2.18 \cdot 10^{9}$ & 2.2 & 2.8 & 60 & 16 & $1.2 \cdot 10^{4}$ & 15, left \\
\hline 10 & 08:20 & $2.94 \cdot 10^{9}$ & 2.2 & 2.8 & 65 & 20 & $2.0 \cdot 10^{4}$ & 15 , right \\
\hline 11 & $08: 23$ & $2.84 \cdot 10^{9}$ & 2.0 & 2.7 & 60 & 18 & $1.0 \cdot 10^{4}$ & 16, left \\
\hline 12 & $08: 24$ & $2.63 \cdot 10^{9}$ & 1.8 & 1.9 & 100 & 20 & $1.0 \cdot 10^{4}$ & 16 , right \\
\hline 13 & $08: 26$ & $3.03 \cdot 10^{9}$ & 1.7 & 1.8 & 90 & 18 & $1.5 \cdot 10^{4}$ & 17, left \\
\hline 14 & $08: 27$ & $2.87 \cdot 10^{9}$ & 1.5 & 1.6 & 80 & 20 & $2.0 \cdot 10^{4}$ & 17 , right \\
\hline 15 & $08: 29$ & $2.87 \cdot 10^{9}$ & 1.4 & 1.5 & 80 & 10 & $1.1 \cdot 10^{4}$ & 18, left \\
\hline 16 & 08:31 & $2.95 \cdot 10^{9}$ & 1.3 & 1.5 & 70 & 6 & $0.7 \cdot 10^{4}$ & 18 , right \\
\hline
\end{tabular}




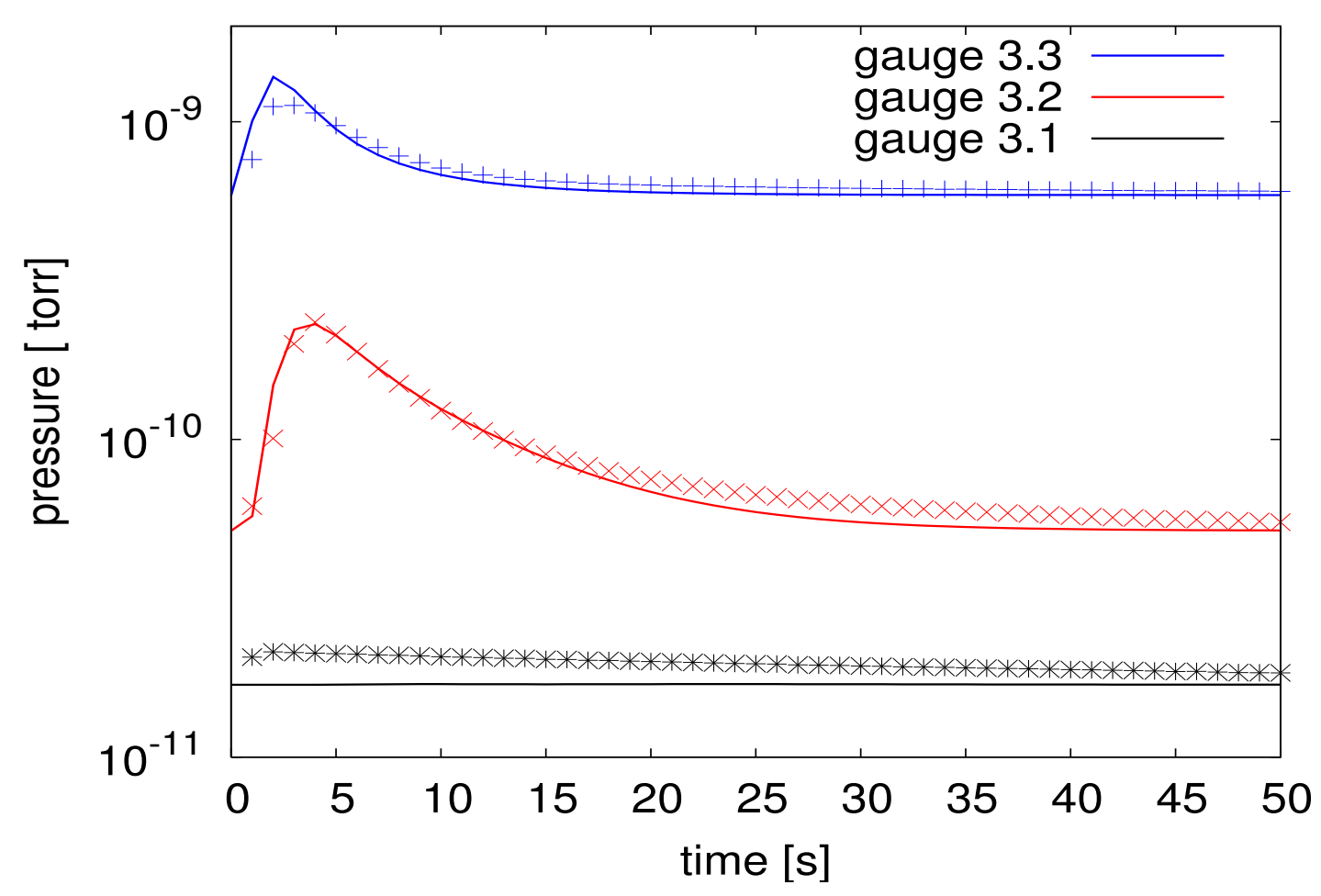

Figure 8: Measured vacuum pressure evolution (blue and red points) and reproduced behaviour using Eq. 10 introducing a gas with two components. The agreement using the parameteres shown in Table 2 is very good.

\section{A simple method to analyze the pressure bumps}

As stated earilier in the text, although time response measurements show the pressure gauges have a delay $(\approx 100 \mathrm{~ms})$, which does not influence the pressure readings, there is still the possibility that a significant delay can be produced by the capacitance created by the large length of the cables (from the tunnel to service building). This capacitance test is planned to be carried out as soon as possible.

An efficient and simple method to obtain the desorption coefficient is considering the areas under the pressure bump. The advantage is that this area (in units of torr $\cdot s$ ) multiplied by the pumping speed (in $l / s$ ) is directly the amount of extra gas (in torr $\cdot l$ ) pumped by the adjacent pump as consequence of the pressure excursion. This is independent of any gas-conserving mechanism such as long sticking times, or of signal low-pass filtering which may slow down the pulse to eliminate noise (see Fig. 9). The presence of such a filters in our system is uncertain. In this case, the desorption coefficient is

$$
\eta_{e f}=G \frac{d P_{i} \cdot d t_{i} \cdot S_{e f f}}{N_{b}}
$$

where $G=3.3 \cdot 10^{10} l \cdot$ torr is just a constant to convert the pressure into molecules, and $N_{b}$ is the intensity of the beam dumped into the wall.

Using this method, the results are shown in Fig. 10. We should note that the areas for the pw3.1 and pw3.2 gauges are fairly accurate because the pulses are short enough for rather accurate base-line subtraction, while this is not quite the case for pw3.3. In this last case the numbers shown are somewhat underestimated, and a more sophisticated deconvolution of the peaks would be desirable. Even better, longer intervals between measurements would be preferable for future experiments.

We see that the yields values are roughly similar to the ones obtained with the pulse shape fitting approach. In particular, disregarding again the 2 first pressure bumps (which we assume they hit close to the polarimeter), the average desorption coefficient in this case is $\eta_{e f}=2.7 \pm 1.5 \times 10^{4}$ molecules/ion. There are however features of both results that cause some concern. Note for example, that the yields do not 

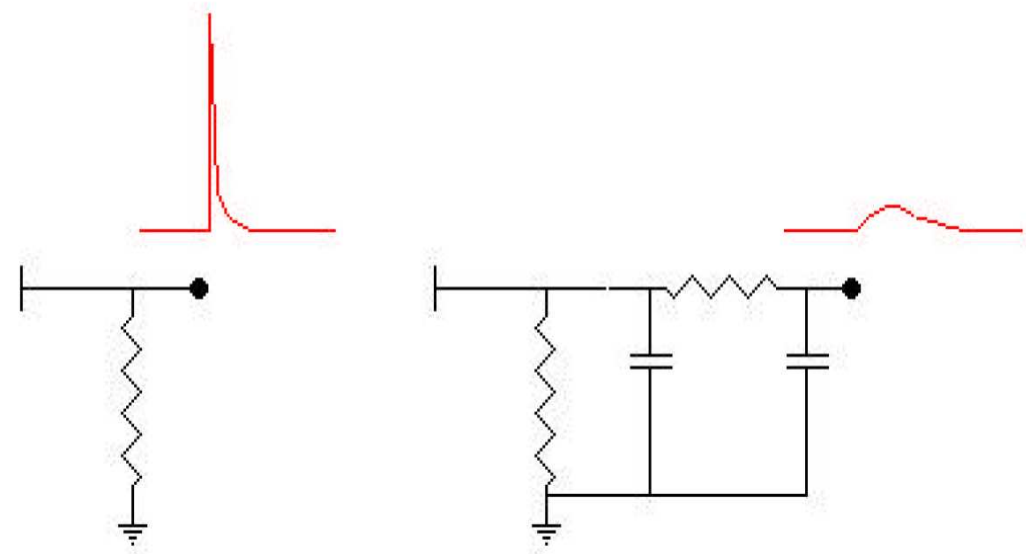

Figure 9: The low-pass filter shown to the right conserves the area under the peak.

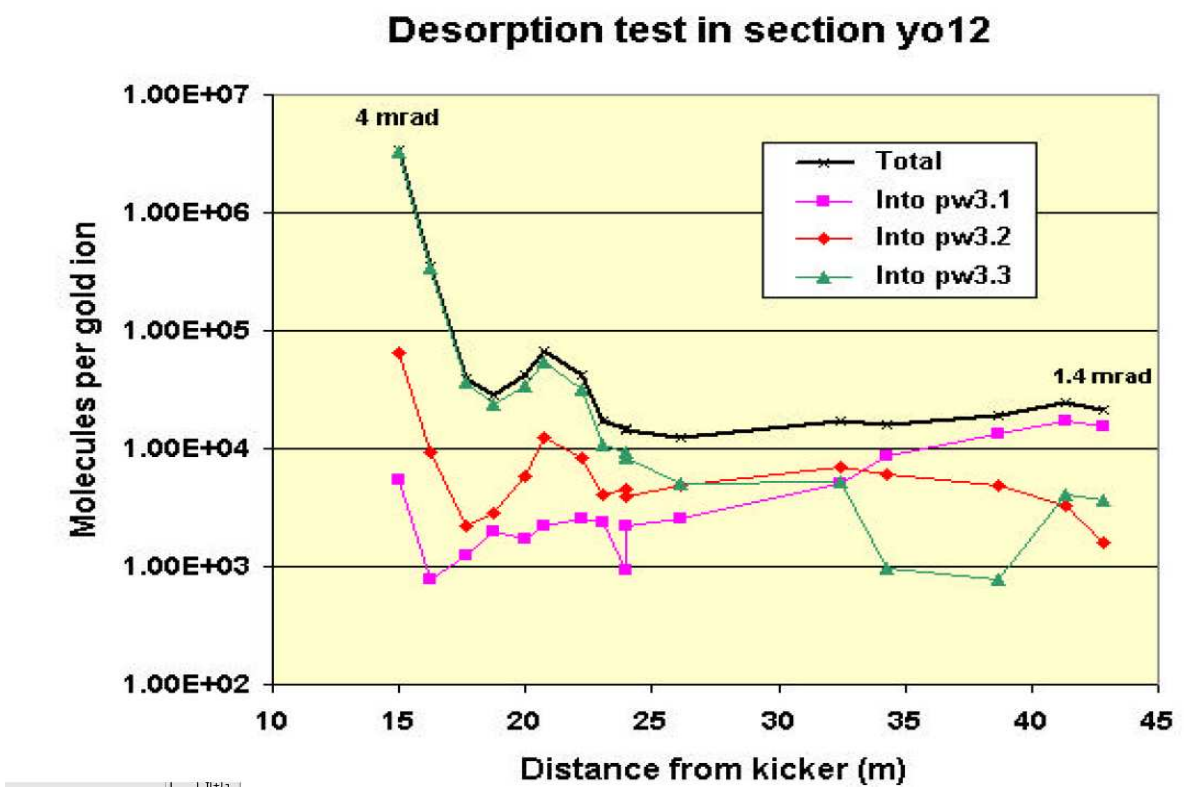

Figure 10: Partial and total desorption yields calculated by using the areas under the pressure peaks.

increase much, as the angles get smaller. From $4 \mathrm{mrad}$ to $1.4 \mathrm{mrad}$ one would expect a factor of 3 increase. We suspect error bars on the measurements (precision of orbit display, pressure gauge readings, and beam intensity) do not allow to notice this effect.

\section{Conclusion}

A set of 16 shots of gold ions $(Z=79+)$ dumped onto the pipe wall in section "yo12" has been analyzed to obtain desorption coefficients of high energy $(10 \mathrm{GeV})$ gold ion beams at grazing angles. The calculations show that desorption coefficient for that surface are around $3 \cdot 10^{4}$ for angles between 1 and $4 \mathrm{mrad}$, consistent with the observations at the CERN test-stand [8], and in the SIS synchrotron of GSI [6], although that results refer to lower beam energies. The results agree also with the analysis done using the well known method of the areas. In order to completely rule out the possibility that the pressure readings are responsible for the observed delays, the effect of the capacitance induced by the cables length is going to be tested shortly. 
Since the cable length is not responsible for such a delays, the dynamic evolution of the pressure bumps can only be reproduced with the introduction of additional surface parameters. The slow time growth of the pressure bumps is explained by the introduction of the surface resident time $(\tau)$ of the molecules, while slow decay is explained by the influence of the linear pumping speed of the beam pipe walls and the different types of molecules. Unfortunately, the experiment was performed in an area which does not have Residual Gas Analyzers, therefore the influence of the different gases cannot be determined. As a future outlook, the measurements carried out in other sections will be analyzed to constrain the results and confirm the reproducibility of this study.

\section{Acknowledgements}

We are very grateful for discussions with, and support from, M. Blaskiewicz, A. Drees, W. Fischer, S. Peggs, R. Tomás, L. Wang, and J. Wie (BNL); and I. Hofmann, O. Boine-Frankenheim and C. Bellachioma (GSI); and J.-M. Laurent (CERN).

\section{References}

[1] Commissioning of RHIC Deuteron-Gold Collisions, T. Satogata et al. TPPB043, Proceedings of PAC'03, Portland, May 2003.

[2] RHIC Pressure Rise and Electron Cloud, S.Y. Zhang et al. MOPA010, Proceedings of PAC'03, Portland, May 2003.

[3] Performance of RHIC beam vacuum system during high intensity operation., H.C. Hseuh at the $8^{\text {th }}$ European Vacuum Congress, held at Berlin, Germany, June 2003.

[4] Final Summary report of Working Group 1.: Electron and Ion Desorption., E. Mahner et al at the $13^{\text {th }}$ ICFA Beam Dynamics Mini-Workshop on "Beam Induced Pressure Rise in Rings", held at Brookhaven National Laboratory, Upton, NY, December 2003.

[5] CERN Accelerator School on Vacuum Technology, editor S. Turner, CERN 94-01, Vol.II, pp. 717-730.

[6] A theory of the beam loss-induced vacuum instability applied to the heavy-ion synchrotron SIS18, E. Mustafin, O. Boine-Frankenheim, I. Hofmann, H. Reich-Sprenger, P. Spiller. NIM A 510 (2003) pp.199205 .

[7] Foundations of Vacuum Science and Technology editor J.M. Lafferty, John Wiley And Sons Inc., New York, 1998.

[8] Molecular desorption of stainless steel vacuum chambers irradiated with $4.2 \mathrm{MeV} / \mathrm{u}$ lead ions Mahner, E; Hansen, J; Laurent, J M; Madsen, N. PRST-AB 6, (2003) 013201.

[9] High-vacuum technology. A practical guide Marsbed H. Hablanian, Ed. L.L. Faulkner, 2nd Edition, Marcel Dekker, Inc, New York, 1997. Ch. 2, pg 27-28, 322.

[10] The dynamic behaviour of pressure bumps in the ISR O. Gröbner. CERN/ISR-VA/76-25, 1976.

[11] Pressure equations for the residual gases in an Ultrahigh Vacuum system, J.P. Hobson and J.W. Earnshaw, J. Vac. Sci. Tech., vol.4, no. 5. 1967. 

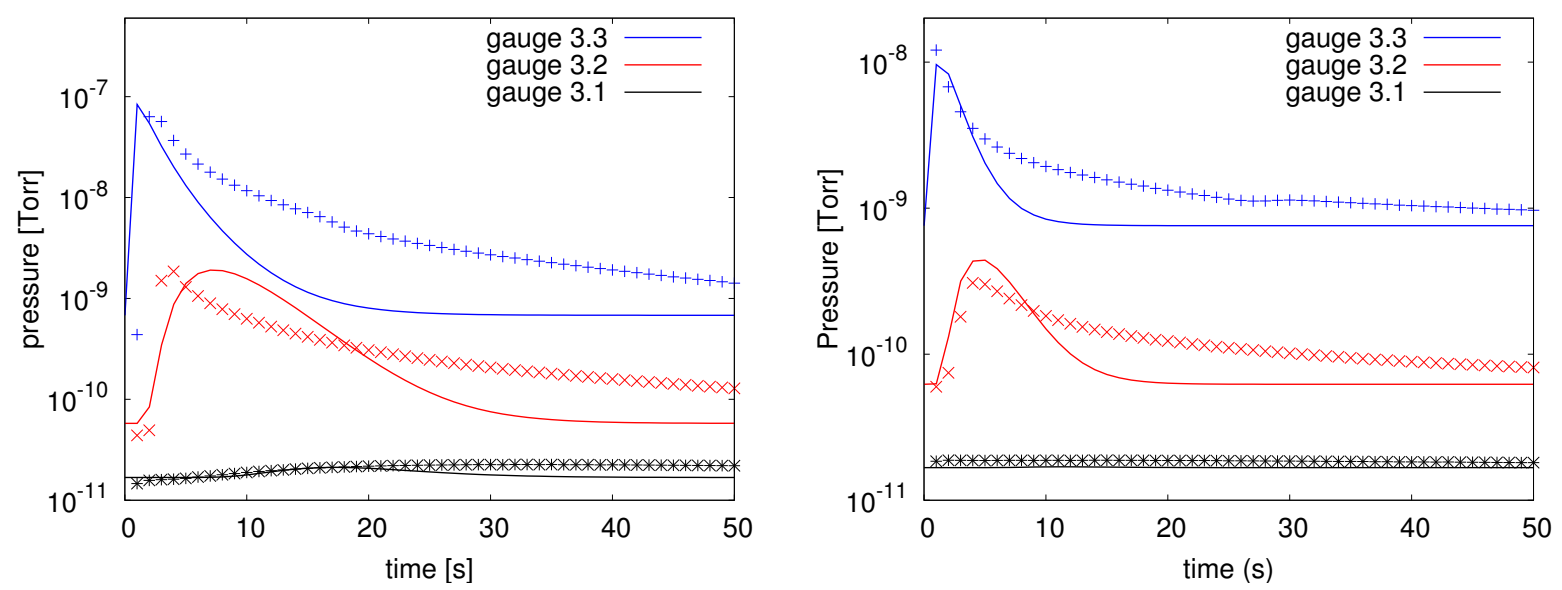

Figure 11: Pressure bump \#1 (left) and \#2 (right). The beam hits the polarimeter region and creates higher pressure rises than in the rest of the beam pipe.
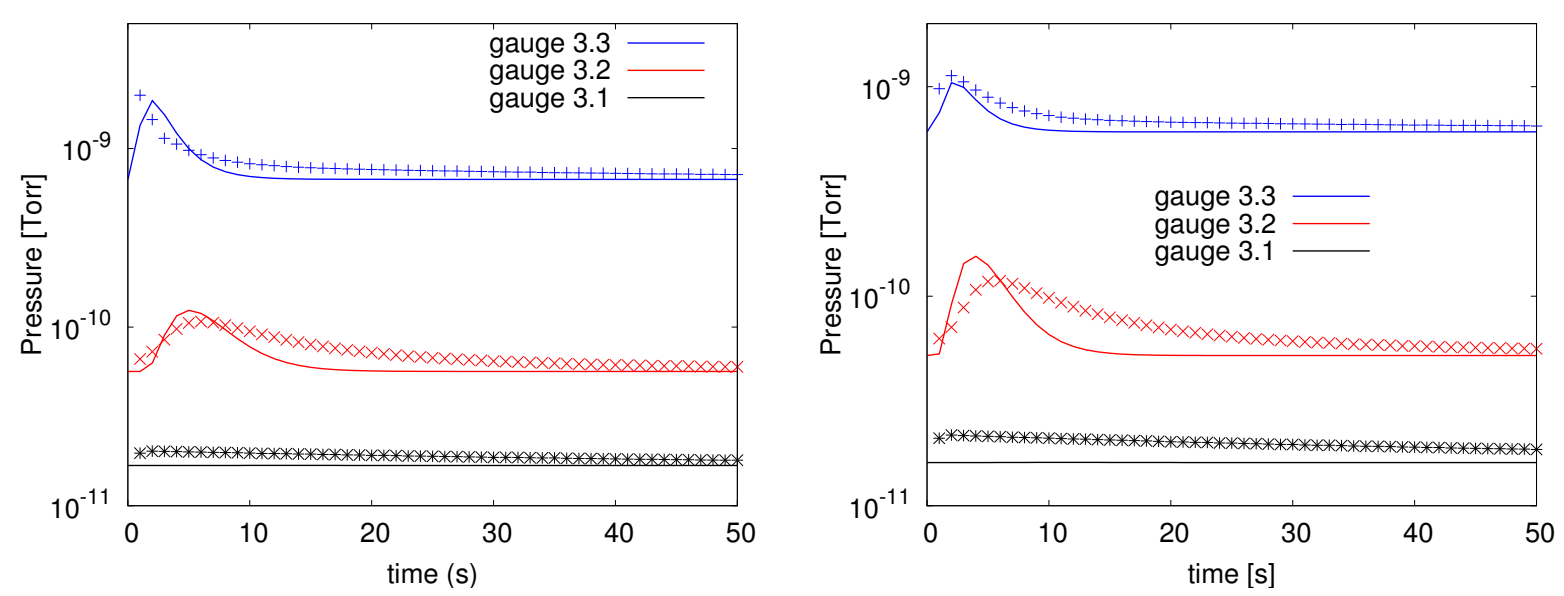

Figure 12: Pressure bump \# 3 (left) and \# 4 (right). 

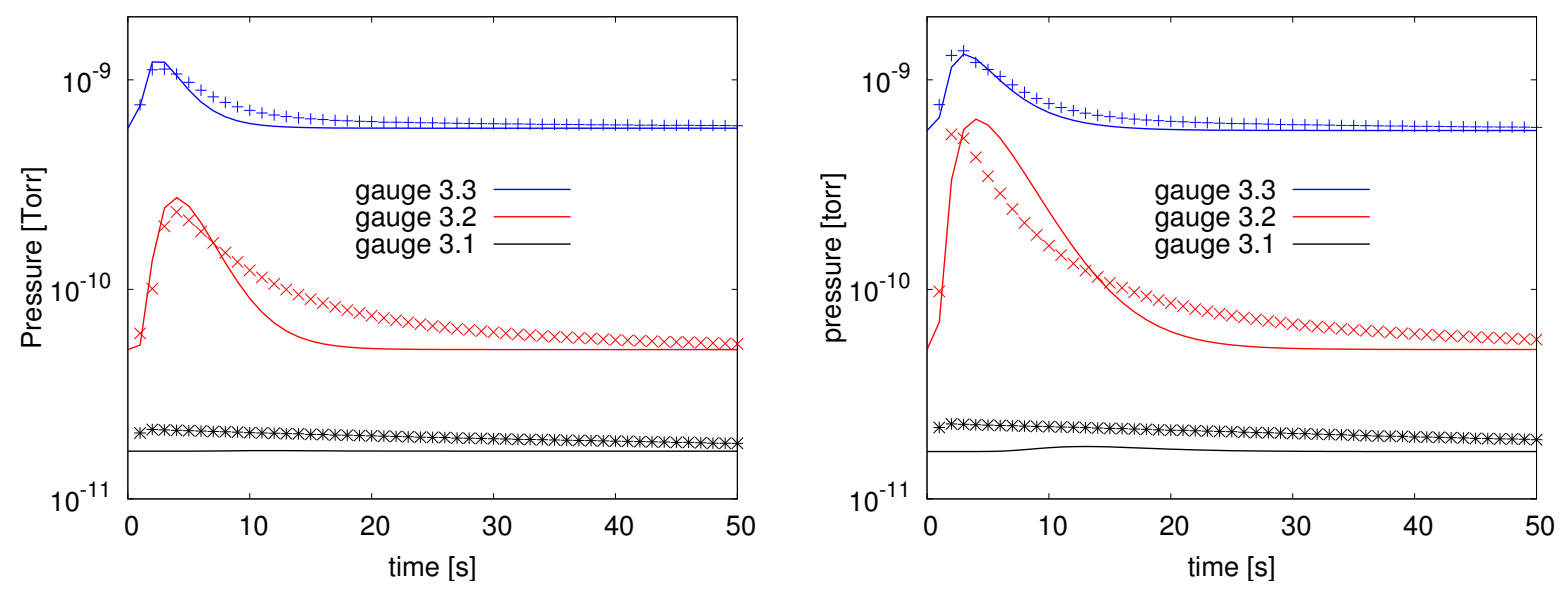

Figure 13: Pressure bump at \# 5 (left) and \# 6 (right).
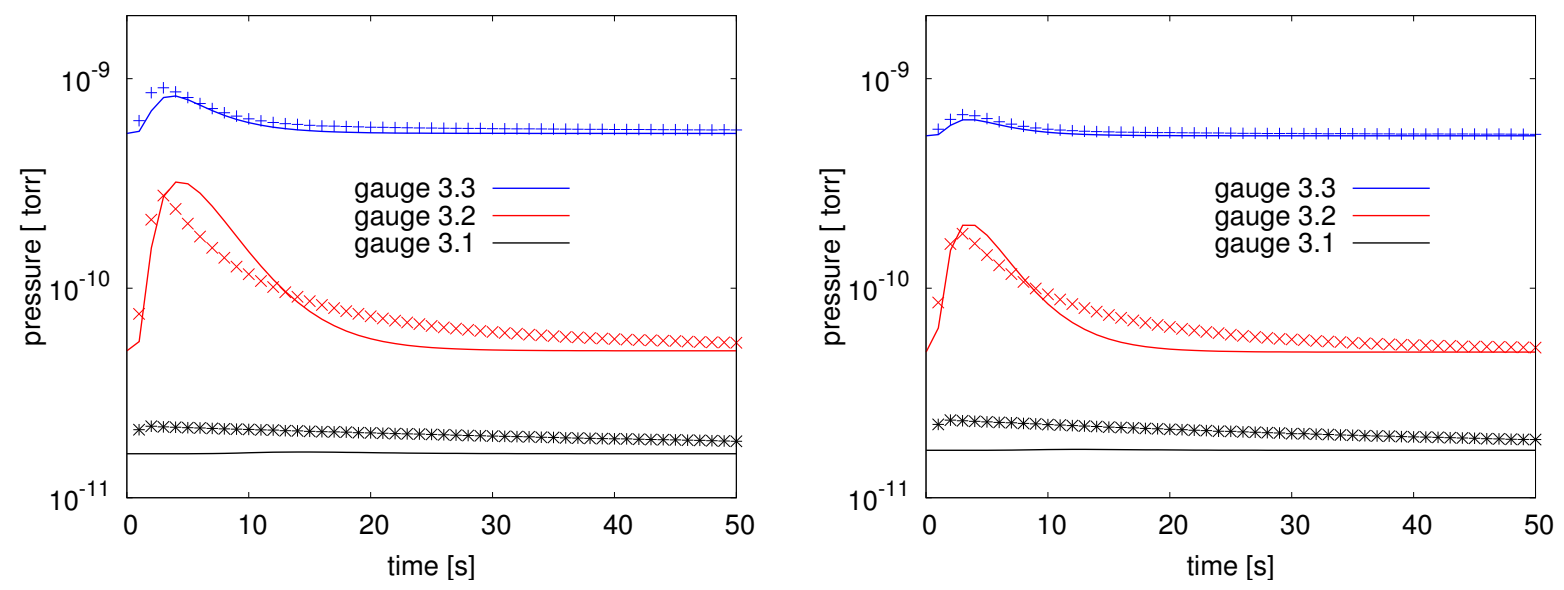

Figure 14: Pressure bump at \# 7 (left) and \# 8 (right).
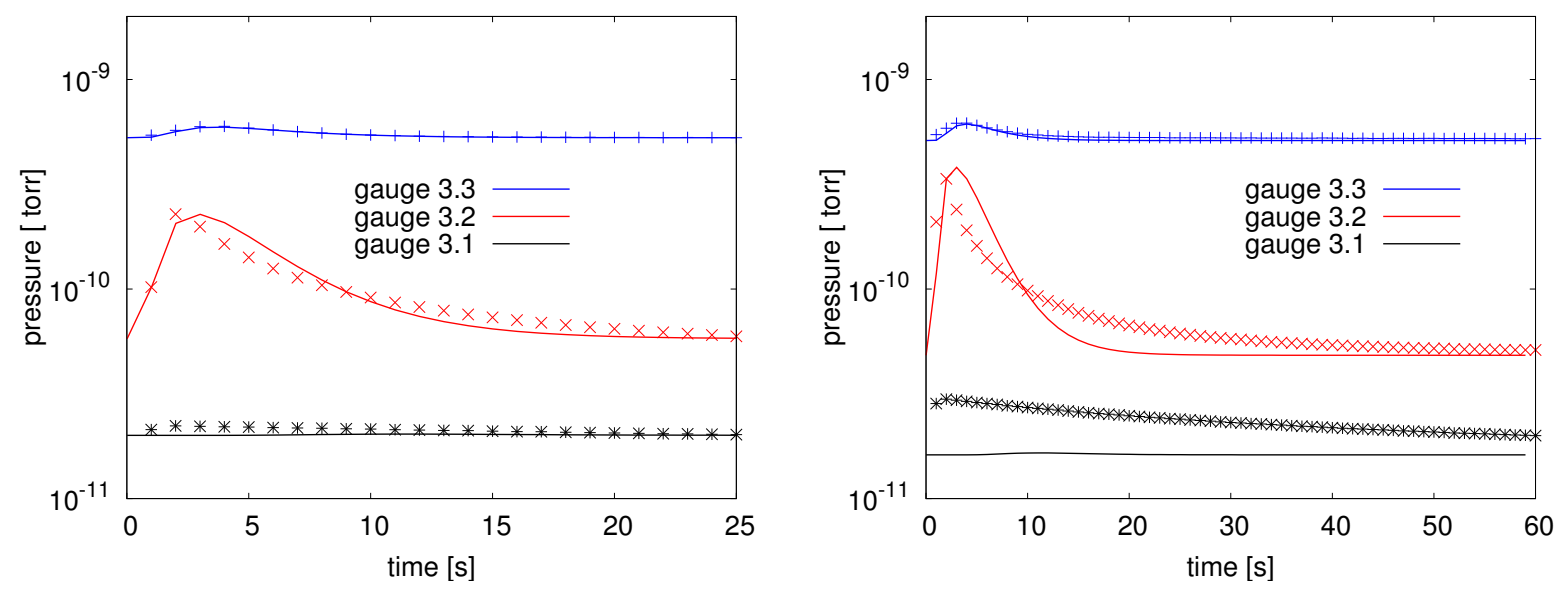

Figure 15: Pressure bump at \# 9 (left) and \# 10 (right). 

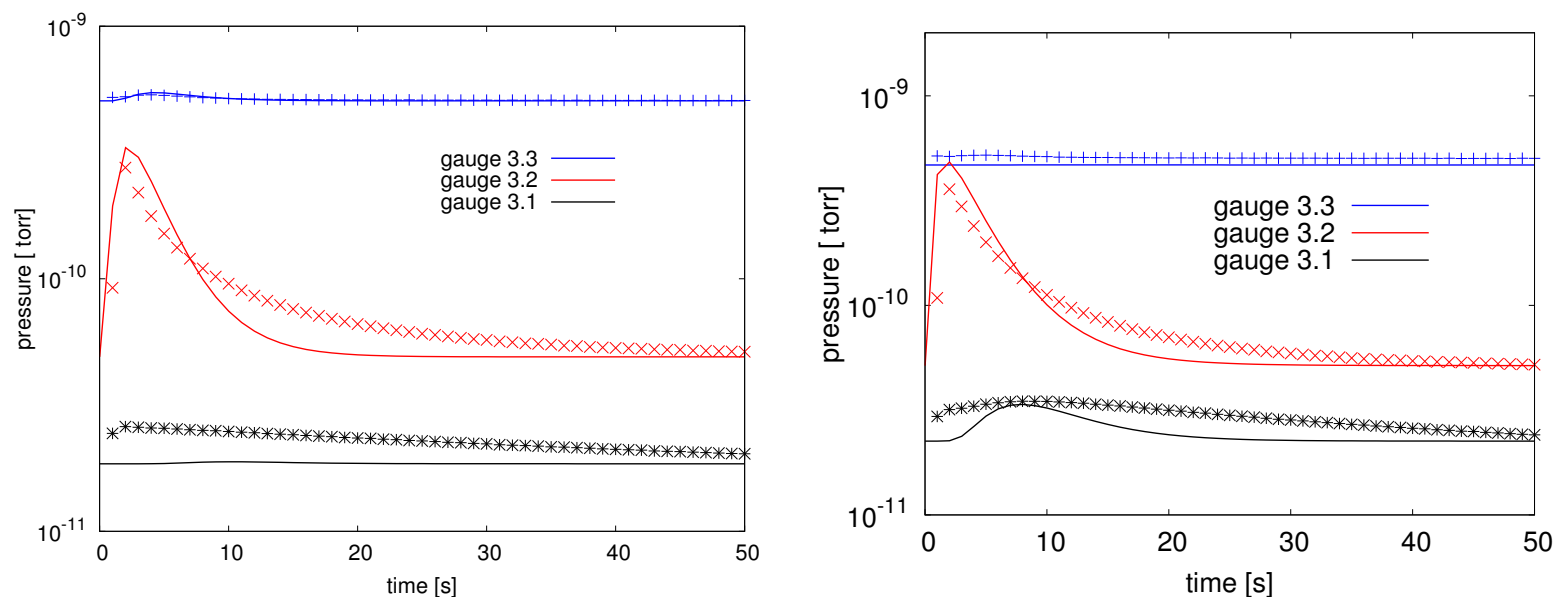

Figure 16: Pressure bump at \# 11 (left) and \# 12(right).
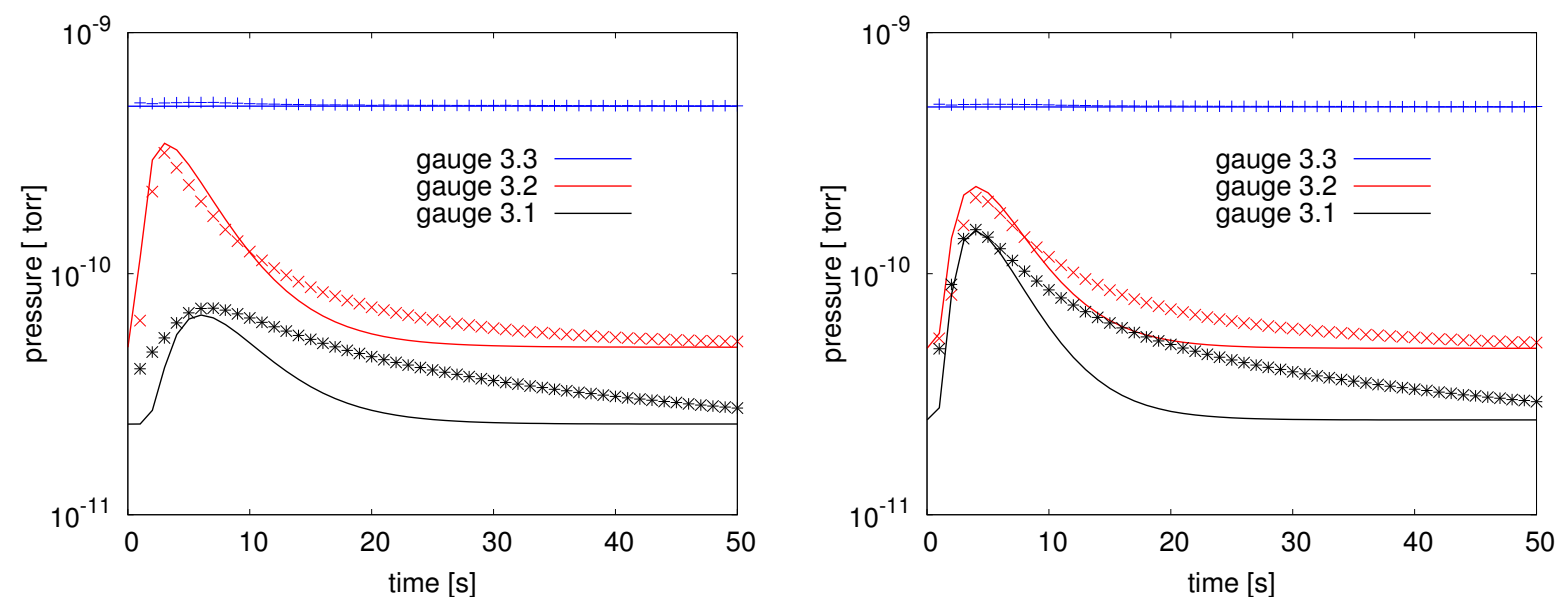

Figure 17: Pressure bump \# 13 (left) and \# 14 (right).
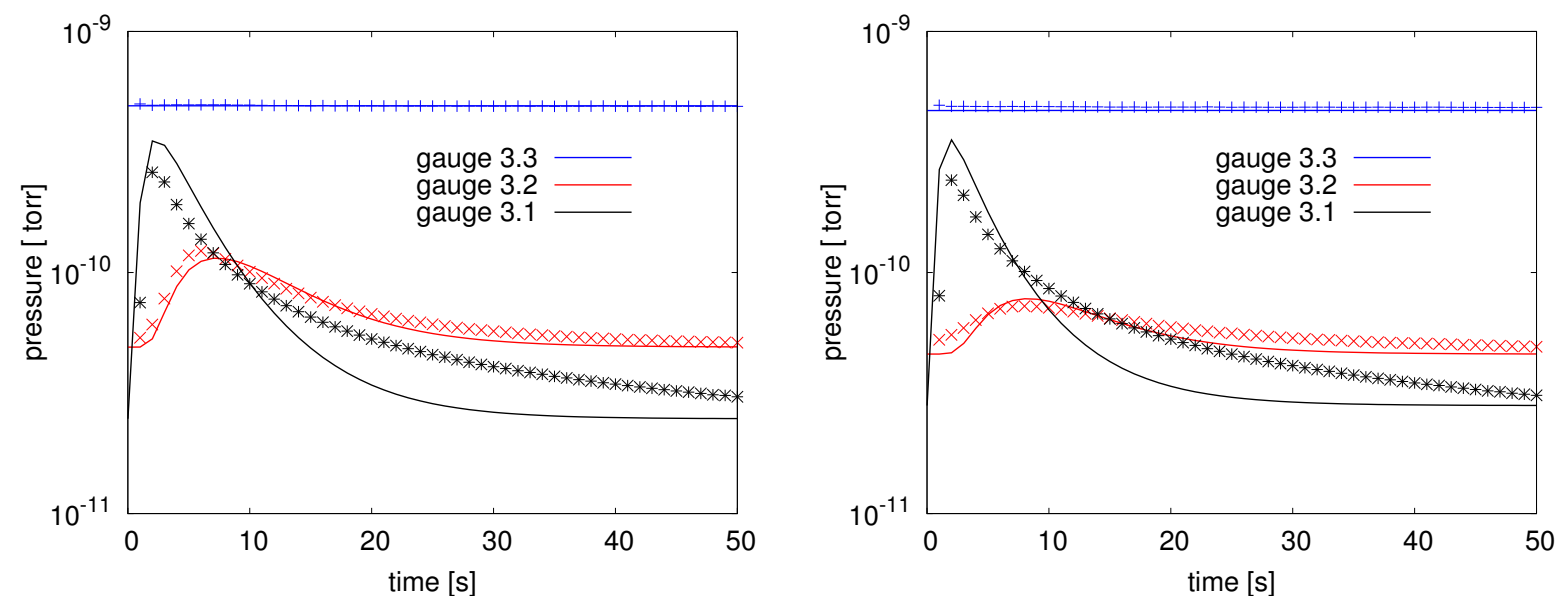

Figure 18: Pressure bump \# 15 (left) and \# 16 (right). 